\title{
LKB1 as the ghostwriter of crypt history
}

\author{
Marnix Jansen • Danielle Langeveld • \\ Wendy W. J. De Leng • Anya N. A. Milne • \\ Francis M. Giardiello • G. Johan A. Offerhaus
}

Published online: 30 July 2011

(c) The Author(s) 2011. This article is published with open access at Springerlink.com

\begin{abstract}
Familial cancer syndromes present rare insights into malignant tumor development. The molecular background of polyp formation and the cancer prone state in Peutz-Jeghers syndrome remain enigmatic to this day. Previously, we proposed that Peutz-Jeghers polyps are not pre-malignant lesions, but an epiphenomenon to the malignant condition. However, Peutz-Jeghers polyp formation and the cancer-prone state must both be accounted for by the same molecular mechanism. Our contribution focuses on the histopathology of the characteristic PeutzJeghers polyp and recent research on stem cell dynamics and how these concepts relate to Peutz-Jeghers polyposis. We discuss a protracted clonal evolution scenario in PeutzJeghers syndrome due to a germline LKB1 mutation. Peutz-Jeghers polyp formation and malignant transformation are separately mediated through the same molecular mechanism played out on different timescales. Thus, a single mechanism accounts for the development of benign Peutz-Jeghers polyps and for malignant transformation in Peutz-Jeghers syndrome.
\end{abstract}

Keywords LKB1 - Peutz-Jeghers syndrome - Hamartoma $\cdot$ LGR5 · Clonal evolution · Polyp

M. Jansen ( $\square)$ · G. J. A. Offerhaus

Department of Pathology, Academic Medical Center, PO Box 22660, 1100 DD Amsterdam, The Netherlands e-mail: M.Jansen@amc.uva.nl

D. Langeveld - W. W. J. De Leng - A. N. A. Milne ·

G. J. A. Offerhaus

Department of Pathology, University Medical Center, Utrecht, The Netherlands

F. M. Giardiello

Department of Medicine, Johns Hopkins University School

of Medicine, Baltimore, MD, USA

\section{Introduction}

In his original monograph 'On the remarkable familial aggregation of mucosal polyposis of the gastrointestinal and oropharyngeal tract and a pigmentation disorder', Dr. Jan Peutz explains how he first traced this constellation of peculiar abnormalities in one family. His case description, published in 1921 in a monthly Dutch medical journal, details the clinical vignette of an adolescent boy presenting to the department of surgery of his hospital in The Hague with symptoms of intestinal obstruction. Emergency laparotomy revealed an obstructing polyp in the small bowel which was excised, after which the boy made a complete recovery (Fig. 1). Previously, on routine physical exam, Dr. Peutz had noticed perioral pigmentation in his young patient. Moreover, on rigid rectoscopy numerous polyps, akin to the one observed during emergency laparotomy, had been found. The remarkable perioral pigmentation in his index patient reminded Jan Peutz of a similar-looking pigmentation abnormality he had seen in a patient referred to him 1 year previous. It turned out that this last patient was in fact the younger brother of his index patient. In his case description, Dr. Peutz recalls how it was the mother of his index patient who pointed out that 'these peculiar pigmentations only seemed to occur in the children that resembled their father'. We can only assume that this comment triggered dr Peutz to examine the other family members. Subsequently, he describes how similar abnormalities were found in four siblings of his initial patient.

Ninety years after his original publication we know that the patients described in his report were affected by a cancer-prone polyposis syndrome that bears Peutz's name. We have continued to follow this original family [1]. Germ-line sequencing of members of this pedigree revealed a $\mathrm{T}$ insertion in exon 1 at codon 66 (535insT) that 


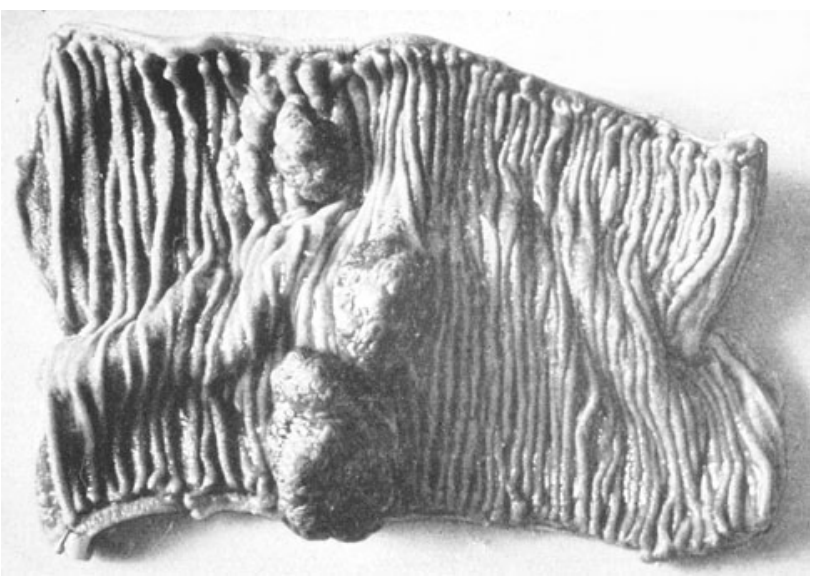

Fig. 1 Depiction of the original small bowel resection specimen containing the PJS polyp that led to an episode of small bowel obstruction in the index patient first described by Jan Peutz in his monograph on the pedigree

resulted in a frameshift producing a stopcodon in exon 4 (codon 162) of the LKB1 gene. Pedigree analysis reveals that of the original family only 17 of 22 affected family members survived into adulthood. The mean age of death for affected family members was substantially younger than for unaffected family members (38 vs. 69 years) and causes of death included bowel obstruction, and intestinal and extraintestinal cancers. This family testifies that PeutzJeghers syndrome (PJS) is not a benign disorder and encapsulates the major clinical areas of concern in a nutshell.

Although much has been learned about Peutz-Jeghers syndrome, many questions still remain. This issue brings together many fascinating points of research all revolving around Peutz-Jeghers and the germline genetic defect. My contribution (as a surgical pathologist) deals with two aspects, the histopathology of the characteristic Peutz-Jeghers polyps and, secondly, recent research on stem cell dynamics and how this relates to Peutz-Jeghers polyposis.

\section{Polyp histopathology: 'naming and shaming'}

Familial cancer syndromes present rare insights into malignant tumor formation. Syndromes such as familial adenomatous polyposis (FAP) are characterized clinically by the development of large numbers of neoplastic precursor lesions some of which, due to their sheer number, will progress to cancer. Whereas in syndromes such as Lynch syndrome (previously, known as hereditary nonpolyposis colorectal cancer or HNPCC) only few precursor lesions develop, most of which are guaranteed to progress to cancer. PJS is characterized by the development of histologically distinct gastrointestinal polyps. Since patients with PJS are at an increased risk of developing cancer in the gastrointestinal tract, it is important to establish whether these polyps carry an inherent risk of neoplastic transformation. As both the polyps and the cancer-prone condition are due to a germline mutation in the LKBI tumor suppressor gene, an understanding of the mechanism of polyp formation should reveal important insight into the cancerprone condition of these patients; regardless of whether the polyps per se are ultimately deemed to lack an inherent risk of transformation.

The first sign of PJS is a distinctive pigmentation observed around the lips, oral mucosa, genitalia or palmar surfaces appearing early in childhood [2, 3]. However, gastrointestinal polyps cause the main clinical symptoms. These may develop throughout the gastrointestinal tract, grow to large sizes, and cause obstruction, pain, and gastrointestinal bleeding with anaemia [4]. A polyp is defined as any abnormality that protrudes above the mucosal surface. Polyps are further subclassified by detailed microscopic analysis of their mucosal lining and any associated histological features. Consequently, polyposis syndromes are primarily classified by the histological appearance of the polyps and further subclassifications can be made by analzying phenotypic expression, systemic manifestations or the exact underlying genetic cause. For example, FAP patients display adenomatous polyps which may harbour varying grades of dysplasia or invasive carcinoma. The large gastrointestinal polyps typically encountered in PJS, are pedunculated polyps with non-dysplastic overlying epithelium (Fig. 2). A characteristic feature of mature PJS polyps is the prominent core of arborizing smooth muscle, which extends into the head of the polyp. Smooth muscle proliferation appears to accompany epithelial hyperproliferation during the earliest stages of polyp development [5]. Of note, smooth muscle proliferation is not a histopathological feature specific for the PJS polyp. For example, PJSlike smooth muscle proliferation can also be observed in conventional sporadic adenomatous polyps. In this setting, the smooth muscle proliferation develops secondary to mechanical insults (due to intestinal peristalsis) during adenomatous polyp growth (Fig. 3) [5]. Furthermore, other conditions unrelated to PJS such as mucosal prolapse syndrome may present with histological features similar to those observed in PJS polyps [5, 6]. Thus, it is unclear whether the observed smooth muscle proliferation is causally involved in polyp initiation or, alternatively, develops only secondarily to accommodate epithelial hyperproliferation. We return to this point below.

The potential for malignant degeneration of PJS polyps and, specifically, whether cancer in PJS patients arises from the non-dysplastic epithelium covering PJS polyps remains a hotly debated subject. Analogous to the genetic lessons learned from the stepwise tumor progression 


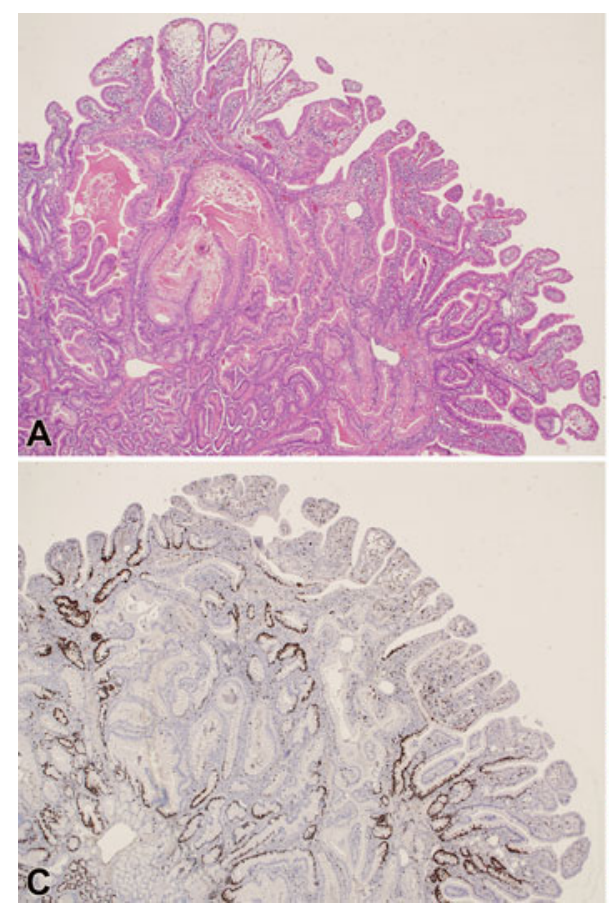

Fig. 2 Overview of histological findings in a characteristic PJS polyp, in this case resected from the duodenum. A H\&E staining showing an overview of the polyp. The epithelial lining demonstrates normal gobletcel-bearing epithelium without signs of dysplasia. Reactive epithelial changes can be noted at the surface of the polyp, which shows edematous villi and active inflammation, presumably due to surface erosion. Towards the center some crypts contain inspissated mucin. b Smooth muscle actin (SMA) immunohistochemical staining

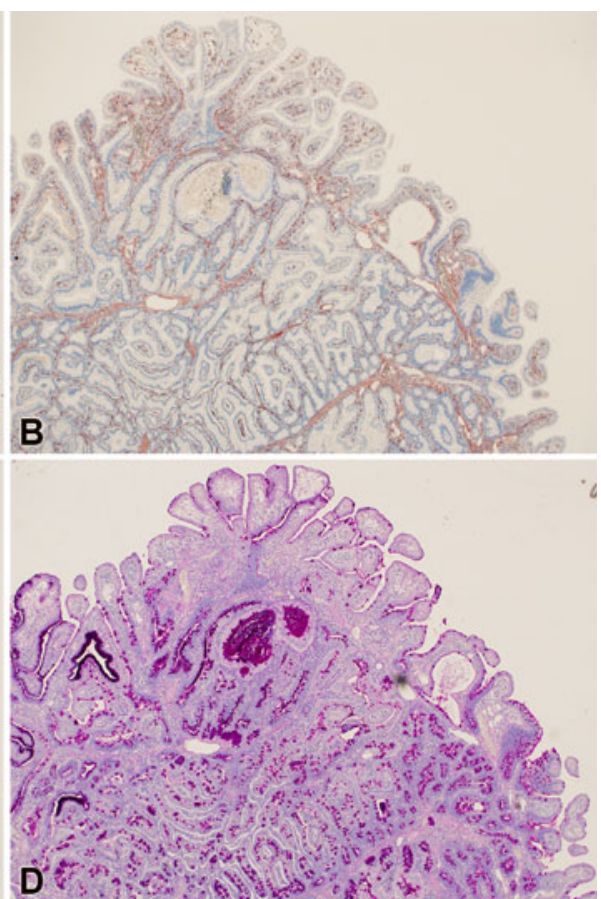

showing the arborizing core of smooth muscle (labeled red) that characterizes PJS polyps. c Ki67 proliferation marker immunohistochemical staining showing that proliferation is restricted to the crypt bases indicating proper compartimentalization of proliferation and differentiation. d Periodic-acid Schiff stain after diastase digestion showing normal differentiation and maturation of the secretory goblet cell lineage
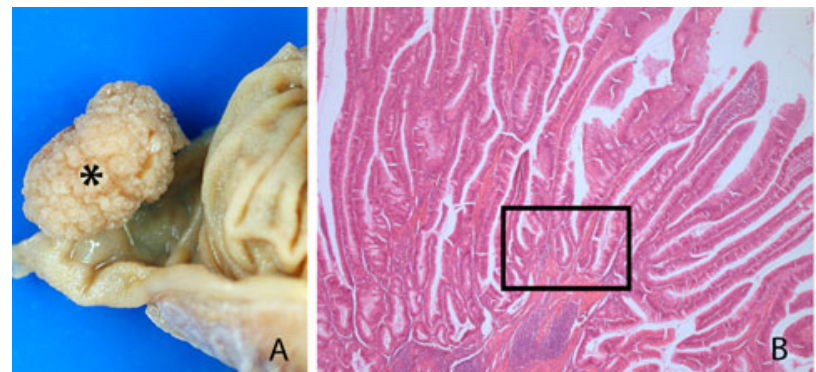

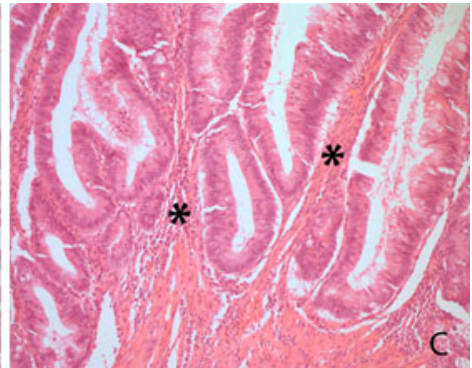

polyp. Note the slender extensions of the smooth muscle core into the tips of the villous projections (indicated by asterisk in c). This arborizing core of smooth muscle resembles the histology shown in Fig. $2 \mathrm{~b}$ for the polyp removed from the duodenum of a PJS patient. c Unlike PJS polyps, which are characterized by a non-dysplastic hyperproliferative epithelial lining, this polyp has a dysplastic epithelial covering classified as low-grade dysplasia. The boxed area in $\mathbf{b}$ is shown at higher power in $\mathbf{c}$

harbour an underlying risk for malignant transformation in spite of their benign appearance? Take the above mentioned case of a conventional adenomatous polyp displaying a prominent PJS-like core of arborizing smooth muscle as an example. It would-on histopathological grounds alone - not be possible to discern whether an adenomatous polyp secondarily acquired a PJS-like smooth muscle stalk 
or, alternatively, a PJS polyp has undergone adenomatous transformation. Historically, case reports have described dysplastic adenomatous transformation occurring in a supposedly pre-existent hamartomatous PJS polyp [7]. However, dysplasia occurring in classical PJS polyps appears to be a very uncommon phenomenon. In series from the renowned familial polyposis registry at St Mark's hospital, a review of 491 PJS polyps showed no evidence of dysplasia in any [8]. Moreover, follow-up over 45 years of 48 patients at the Mayo Clinic failed to reveal dysplastic change in any PJS polyp either [9]. More recently, we proposed that the historical accounts of adenomatous transformation occurring in PJS polyps might be explained by a blurring of the order of events; that is, these lesions likely arose from a conventional adenoma arising in a PJS patient that secondarily developed a PJS-like smooth muscle stalk, akin to the situation encountered in sporadic patients, as described above [5].

Since the focus of cancer initiation in PJS patients remains to be definitively settled, proper histopathological classification of PJS polyps is an important issue. Traditionally, mature PJS polyps have been classified as hamartomas. A hamartoma is defined as a combined and non-dysplastic overgrowth of all tissue layers native to the site of origin of the lesion in equal proportion. However, the 'hamartoma' designation for PJS polyps is presently not based on an etiological rationale and purely descriptive features such as the prominent core of arborizing smooth muscle and epithelial hyperproliferation may lack a common causal relationship as stipulated by the hamartoma designation. The hamartoma classification may, therefore, be misleading (and may ultimately prove to be a misnomer) because the actual etiological mechanism of polyp development remains unclear at this point. This consideration is also relevant to studies that have appeared in literature linking the so-called 'hamartoma syndromes' PJS, tuberous sclerosis and Cowden's disease [10]. Clinically it appears artificial to lump these syndromes together since they share little medical overlap other than the 'hamartoma' designation. Cowden's disease, for example, is characterized by juvenile polyps. Using the designation 'hamartoma' as an all-encompassing term for polyps that currently defy proper etiological classification does not promote understanding of these syndromes.

Further research examining the histological architecture of PJS polyps in closer detail is therefore warranted. For example, the hamartoma designation mandates that the epithelial covering of PJS polyps should display all lines of differentiation normally observed at the site of origin of a PJS polyp in a normal ratio. However, earlier studies noted that regarding the epithelial covering of PJS polyps, some differentiated epithelial lineages may be lost at the expense of other lineages or in favour of a more immature phenotype altogether [11]. Full-blown PJS polyps commonly reveal these alterations; however, the net shift that occurs towards an immature phenotype is not to be interpreted as a sign of premalignant potential per se. This is because the polyps often display many of these reactive changes (such as decreased epithelial maturation and increased proliferation) along with signs of significant trauma, for example mucosal haemorrhage. In an interesting recent study, epithelial maturation defects in $\mathrm{Lkb}^{+/-}$ murine gastric mucosa was investigated before the onset of frank polyposis [12]. Lina Udd and co-workers show that foci of otherwise unremarkable gastric antral mucosa in $\mathrm{Lkb1}^{+/-}$mice displayed subtle defects in epithelial maturation. Curiously, these minute foci were often associated with Ki67-positive gland bases [12], a point we will return to shortly. Thus, it appears prudent to refer to the gastrointestinal lesions in PJS simply as 'PJS polyps' rather than hamartomas, since the latter would imply an etiological understanding currently not justified. Most importantly, because the malignant potential of characteristic PJS polyps (those polyps demonstrating a prominent smooth muscle core with normal non-dysplastic overlying epithelium) remains uncertain, PJS polyps should be separated from (other) precursor lesions occurring in PJS patients or engineered Lkb1 mouse models [13]. Accurate classification of PJS polyps thus awaits an elucidation of the sequence of events during PJS polyp initiation and the pathophysiological mechanism responsible for PJS polyp development.

To this point we have considered proper histopathological classification of the polyps encountered in PJS patients. Now we focus on recent research involving the LKB1 tumor suppressor gene and how this might help elucidate the mechanism of polyp development in PJS patients. We will examine research, including our own unpublished work that has investigated the role of LKB1 in intestinal stem cell compartments.

\section{Crypt dynamics in Peutz-Jeghers polyposis}

Lineage competition in the gastrointestinal tract

Most epithelial tissues self-renew throughout adult life owing to the presence of multipotent stem cells and/or unipotent progenitor cells [14]. The cells of the intestinal epithelium are arranged hierarchically, becoming progressively more differentiated with age and passage along the crypt-villus axis. The relatively straightforward histological set-up and the high proliferative rate has made the mammalian intestinal stem cell compartment one of the most studied of all mammalian stem cell niches [15]. Recently, through the identification of LGR5 as a murine 


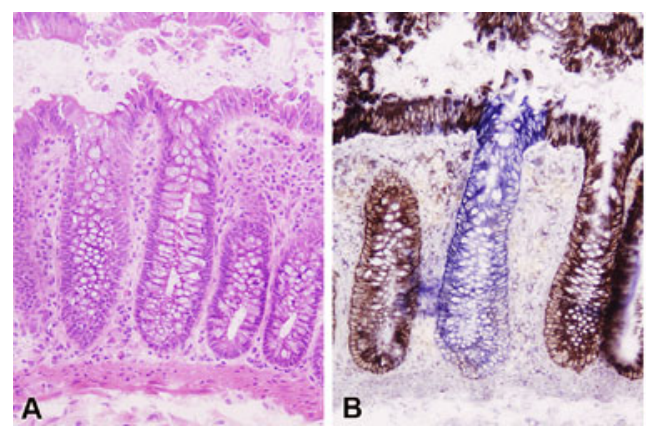

Fig. 4 COX-SDH enzyme histochemistry on normal colon reveals clonal evolution. The dynamics of clonal evolution can be traced by analyzing a polymorphic marker. Owing to an environment with elevated production of free radicals by the respiratory chain and limited repair mechanisms, the mitochondrial DNA (mtDNA) is prone to accumulating mutations. This accumulation is random and increases with age. Dual enzyme histochemistry can be used to simultaneously detect the mtDNA-encoded cytochrome- $c$ oxidase (COX; complex IV of the respiratory chain) and nuclear DNAencoded succinate dehydrogenase (SDH), a component of complex II of the respiratory chain. Sequential COX and succinate dehydrogenase histochemistry highlights deficiencies in COX enzyme activity,

intestinal stem cell marker, the direct analysis of mammalian intestinal stem cells has become possible [16]. These studies show that each intestinal crypt harbors multiple stem cells that together give rise to all other postmitotic differentiated cells populating the villus. All stem cells generate daughter progeny through continuous selfrenewal, and every individual stem cell and its clonal daughter progeny can be considered to represent one lineage in the crypt. Classic studies performed in chimeric animals carrying a mixture of background marker alleles show that stem cell lineages within the intestinal stem cell crypt compartment undergo so-called lineage competition [17]. That is, at birth every crypt in these animals displays a mosaic (polyclonal) composition of marker alleles. However, within several weeks these crypts become monoclonal, retaining just one of the marker alleles. This mechanism of random loss of some lineages with replacement by others is called lineage competition. Elegant studies employing the aforementioned LGR5 stem cell marker have re-affirmed this notion (vide infra). Lineage competition is expected to continue even after the crypt has reached a monoclonal status with respect to its chimeric background. In other words, after one chimeric background has attained dominance, all daughter lineages of this mother lineage will now undergo lineage competition. In time, as a result of random genetic drift, one daughter lineage will again attain dominance, and its daughter lineages will then undergo lineage competition. This is an ongoing cycle of lineage competition within the crypt and this lottery-like process of clonal evolution occurs at steady state in each of the approximately 15 million crypts in the

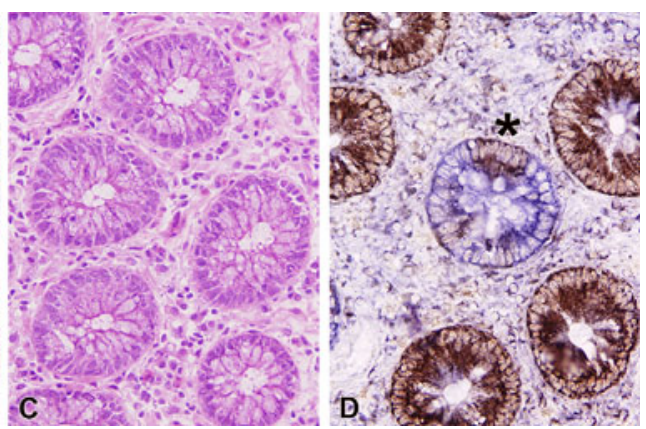

wherein cells lacking COX activity appear blue and normal cells retaining $\mathrm{COX}$ activity appear brown. a, c H\&E staining showing normal mucosa. b Longitudinal section showing a mutation that has occurred in a stem cell lineage that has populated the entire crypt. All cells are COX-deficient (blue). d En face section showing a mutant clone in the process of colonizing the crypt; more than half the cells are now COX-deficient (blue); asterisk indicates clear contrast between COX-deficient (blue) and surrounding nonmutated colonocytes $(\mathbf{a}-\mathbf{d}, \times 200)$. After extinction of all non-mutated lineages, the crypt has been clonally converted. In standard H\&E preparations the dynamic process of lineage competition is obscured from our eyes

adult human colon. The time between two successive stem cell lineages attaining dominance in one crypt is called crypt niche succession time. Importantly, new potential markers such as genomic methylation changes or somatic mutations arise all the time (Fig. 4). However, as no practical method of prospectively identifying and tracing these markers in the human gut exists, the process of lineage competition is obscured from our eyes.

Recently, Hugo Snippert and co-workers for the first time elegantly visualized the behavior of multiple individual lineages 'competing' in the murine intestinal crypt [18]. Their method involved the previously established Brainbow cassette, which recombines in a random fashion to irreversibly label individual stem cells and their future daughters with one of four (green, yellow, red or blue) fluorescent marker proteins. This allows quantification of the number of lineages present over time in individual crypts. Their work firmly establishes lineage competition as the driving force behind clonal evolution. Moreover, it demonstrates that all stem cells in the crypt-and their associated lineages - are equally likely to attain dominance, i.e. all lineages are equipotent. Doug Winton's group in Cambridge employed a different approach to reach a similar conclusion [19]. Both research groups inferred that contrary to previous thinking the dominant mode of stem cell division in the crypt is symmetric, not asymmetric. Note that on a population level, stem cell turnover can be analyzed by tracing the loss (or de novo aquisition) of lineages over time, regardless of the dynamics on the level of the singular stem cell with regards asymmetric or symmetric stem cell division. Other recent 
studies reached a similar conclusion for other stem cell niches, such as the murine epidermal and germ line stem cell compartment $[20,21]$. This body of work indicates that lineage competition in the crypt must be understood by investigating clonal evolution from a population perspective. Fortuitously, previous work has paved the way for investigating this complex matter at the human level.

\section{A population genetics approach to lineage competition}

Studies performed by Darryl Shibata and colleagues in FAP patient material previously formulated a statistical approach to crypt niche succession times [22, 23]. These modeling studies are based on the retrospective analysis of genomic differences within a population of intestinal crypt progenitor cells through the concept of a molecular clock. Molecular clocks, in paleontology, record time between a last common ancestor and species divergence based on the random accumulation of genetic changes according to a pre-determined neutral mutation rate between related genomes [24]. Analogously, it should be possible to reveal phylogenies of progenitor lineages within individual crypts based on the genetic changes accumulated over time since progenitor lineages last went through crypt niche succession [25]. A marker under neutral selection is expected to show an increased measure of diversity as lineages diverge over time. Following this reasoning, Darryl Shibata and his co-investigators analyzed the diversity in methylation patterns of the $\mathrm{CpG}$ island of the CSX gene-which is not expressed in human colon-within and between individual crypts. These crypt heterogeneity patterns were significantly greater in histologically normal FAP crypts compared to crypts from control patients [23]. This indicates that crypt niche succession times are expanded in FAP crypts, since lineages that persist longer retain a greater number of variants.

Prior to the discovery of the energy-sensing AMPK module as a downstream target, LKB1 had been most prominently linked to the regulation of cellular polarization in model organisms. The alleles encoding the nematode and fly counterpart of the LKB1 tumour suppressor were both retrieved in genomic screens designed to pick mutants defective in cellular polarity regulation [26, 27]. Not withstanding the accumulated data on the LKB1-AMPK module in cellular metabolism [28], these early studies remain some of the strongest evidence linking LKB1 to cellular polarization. Later studies in vertebrate model systems provided additional evidence for a role of LKB1 in cell polarity and directed cell division [29]. Consequently, germline LKB1 mutations may upset stem cell turn-over in PJS patients' crypts as well. We, therefore, implemented the technique outlined above involving the analysis of $C S X$ crypt diversity patterns on unaffected PJS mucosa and age-matched control crypts. These analyses are difficult to perform because of the relative scarcity of patient material. Nonetheless, through this unbiased technique, we found that PJS crypts retain a statistically increased number of variants when compared to age-matched controls (unpublished data). Thus, akin to FAP, crypt diversity is greater in unaffected PJS crypts indicating that this physiological process of clonal evolution is protracted in PJS. As discussed below, this protracted clonal evolution scenario implicates a cancer-prone state in unaffected PJS mucosa at normal background mutation rates.

Consequences of a protracted clonal evolution scenario in PJS crypts

Stem cells accumulate mutations stochastically from birth in phenotypically normal epithelia. During life, stem cell populations and their pool of accumulated mutations continuously change as individual lineages become extinct or attain niche dominance. Most mutations in stem cell lineages will be lost, because only one current stem cell lineage attains future dominance [17-19]. These mutations need not evoke a selective advantage and may be initially neutral. Individual mutations without selective advantage may thus initially 'hitchhike' along with the inherent clonal evolution of stem cell niches. The analysis of crypt diversity patterns through neutral markers such as CSX methylation tags is based on this hitchhiking pattern. Eventually though, combinations of mutations confer a visible tumor such as a tubular adenoma. Differences in clonal evolution rate may allow crypt mutations to accumulate at different frequencies even though these mutations arise at a similar rate. This occurs because the rate at which mutations are shed from the crypt depends on the clonal evolution rate [22]. This was also shown in an earlier study comparing crypt-restricted loss of O-acetyltransferase activity between normal colonic epithelia in FAP and Lynch syndrome patients [30]. In this study phenotypically normal FAP epithelium demonstrated more crypts mutant for O-acetyltransferase activity than phenotypically normal age-matched Lynch syndrome epithelium. These findings on $\mathrm{O}$-acetyltransferase activity should extend to other transcripts such as p53 or K-RAS. Thus, in crypts undergoing a protracted clonal evolution scenario such as in FAP [23] or PJS (our unpublished data) genetic clonal diversity is greater.

Differences in the amount of accumulated mutations that persist over time show that the rate of clonal evolution can be protective (or anti-tumorigenic) because mutant stem cells may be lost through lineage competition. Mathematical modelling adapted from population genetics predicts that the chance of loss of novel alleles within a population is given by $\mathrm{p}_{\text {loss }}=(2 \mathrm{~N}-1) / 2 \mathrm{~N}$, where $\mathrm{N}$ is population size and the 
chance of fixation of new alleles is $p_{\text {fix }}=1-p_{\text {loss }}=1 / 2 \mathrm{~N}$. In a similar fashion one can derive that the rate of drift (loss or fixation of novel alleles under neutral selection) is inversely proportional to population size (see Table 1 on the dynamics of surnames in Korea). Therefore, population size has a domineering effect on the rate of fixation or loss of novel alleles and this effect becomes more pronounced in smaller populations. Accordingly, with larger population sizes, the time between population bottlenecks or crypt niche succession increases proportionally. A protracted clonal evolution or increased lineage residence time, therefore, decreases the time to cancer presentation by expanding the pool of selectable variants retained over time. Or, put in other words, a protracted clonal evolution will shorten the time to adenoma formation through the increased likelihood of an accidental acquisition of a biallelic gatekeeper mutation. Thus, by definition, demonstration of an expanded progenitor pool in a niche that undergoes lineage competition reflects a cancer-prone state.

LKB1 hemizygosity expands the progenitor zone in unaffected Peutz-Jeghers mucosa

We previously proposed that the 'hamartomatous' polyps observed in PJS are an epiphenomenon to the cancer-prone condition, and that its characteristic smooth muscle core is indistinguishable from histopathological alterations seen in forms of mucosal prolapse [5]. Analysis of murine PJS polyps in $L k b 1$ hemizygous mice established independently in several laboratories convincingly shows that the wild-type allele need not be lost during polyp formation [31-33]. Following our proposal that the polyps are an epiphenomenon to the cancer-prone condition, we are faced with the remarkable situation of a rare polyposis syndrome where polyps and carcinomas occur simultaneously, yet these are not linked in a clinicopathological sense. However, regardless of whether the polyps lack premalignant potential, an understanding of their development will reveal important insight into the co-existent cancer-prone condition, as the molecular mechanism responsible for polyp formation must, under the simplest model, be responsible for the cancer-prone condition in PJS as well.

We previously observed an expanded crypt progenitor zone in normal intestinal mucosa in PJS patients by standard immunohistochemical labeling with the Ki67 proliferation marker [34]. We inferred that germline mutation of $L K B 1$ leads to a defect in the regulation of stem and/or transit amplifying cell turn-over in the mammalian intestine. Importantly, in this study we observed an elongated progenitor tract in the absence of an increased labeling index (that is, the number of Ki67-labeled nuclei divided by the total number of nuclei in the crypt was not significantly different between PJS crypts and matched controls). This shows that, besides the absolute size of the progenitor zone, other basic characteristics of the crypt as a stem cell niche (such as division rate, the pace of maturation and/or differentiation, and niche exit) are unchanged [35]. The observation of an expanded progenitor tract displaying a normal labeling index argues for dysregulation at the level of the intestinal stem cell. Our data derived from the analysis of dynamic CSX methylation patterns in unaffected PJS mucosa are in full agreement with this hypothesis. Definitive analysis of the size of the dedicated stem cell pool in PJS crypts awaits the availability of direct stem cell markers in human tissue.

Table 1 On lineage competition and the extinction of Korean family surnames

\footnotetext{
Clone size distribution of progenitor cell populations can be described by a model using only three parameters: the overall division rate of proliferating progenitor cells (birth), the rate of niche exit (death) and the symmetric division rate of progenitor cells (extinction rate). Models of this kind have a long history dating back to the works of Galton and Watson and Bienayme in the 19th century on the 'extinction' of family surnames. In our case, progenitor cells mimic the 'male' population, each capable of generating precisely two offspring with 'gender' chosen at random. The lineage of any given clone (family surname) may expand by a progenitor cell (father) giving rise to two progenitor cells (sons), whereas a lineage is driven towards extinction by a progenitor cell giving rise to two cells committed to differentiate (daughters). The probability of a progenitor cell labeled at induction (a patriarch) founding a clone (family) of size $n$ at time $t$ post-induction can thus described by a so-called stochastic Galton-Watson process. This shows that although division and migration processes imply a complex time evolution of clone size, the principle characteristics can be inferred from the behaviour of the progenitor cell population alone, which is governed by the symmetric division rate. It follows that although variations in birth (proliferation rate) and death (differentiation) ratios can in principle impact on clone size evolution, this would also necessitate a change in labeling index (compare our data on progenitor zone expansion in PJS crypts [34]). Deviations in the symmetric division rate (i.e. the probability of having two sons) however, expand clone size (family surname) but leave other characteristics unchanged. Indeed, this appears to characterize progenitor cell behaviour in unaffected PJS crypts

Formulating clone size behaviour according to a stochastic Galton-Watson process also contains the implication that, given enough time, all clones (family surnames) eventually become extinct analogous to the process of clonal conversion in crypts. This extinction probability is governed by the symmetric division rate (probability of having two daughters). Korean surname evolution represents an extreme example of stochastic clone extinction. Here around 250 family surnames survive to this day and nearly half the population carries one of three family surnames (that is, either Kim, Lee or Park)
} 
The forming of a Peutz-Jeghers polyp

In PJS crypts the ratio of proliferative over non-proliferative cells remains essentially the same even though more cells (in absolute numbers) are continuously fed onto the villous epithelial carpet [34]. Incipient polyps might be created by stochastic deviations from the normal equilibrium of stem cell divisions. In the study previously discussed by Lina Udd et al. scattered foci of increased Ki67-labeling in unaffected $L k b 1^{+/-}$gastric mucosa were observed [12]. These stochastic bursts of proliferative activity may form small mucosal elevations which may serve as a leadpoint upon which peristalsis can act. Classically, PJS polyps have been described as numbering from a few to several dozens along the entire length of the gastrointestinal tract. A recent study investigating COX-2 inhibition by sulindac analogues in a small cohort of PJS patients necessitated repeated gastroscopies. This study unexpectedly showed that the gastric epithelium in these patients was in fact studded with 'hundreds of small hyperplastic lesions' [36]. Potentially these lesions wax and wane over time (small outpocketings may be extruded or auto-amputated) and never come to clinical attention. In fact, most larger lesions both in the murine system (typically at the level of the pylorus [2]) as well as in the human system (at the level of the descending colon and rectum [1]) occur at sites of greatest mechanical force. This suggests that incipient polyps occur frequently and along the gastrointestinal tract, but a literal tug of war between mucosal elevations and mechanical force would favour a small mucosal elevation to be moulded into a macroscopic polyp. Indeed, an incipient polyp already shares features with mucosal prolapse, in particular smooth muscle proliferation. A small incipient polyp could serve as the leadpoint for further outpouching due to mechanical forces pulling on the nascent polyp. This would culminate in the full-blown macroscopic cauliflower-like lesion with abundant smooth muscle proliferation microscopically. Importantly, in this scenario smooth muscle proliferation is secondary to, and accommodates for, epithelial hyperproliferation. Our observations on epithelial turn-over dynamics in PJS can explain the formation of incipient PJS polyps as small hyperplastic lesions which lack an inherently increased risk of neoplastic transformation.

\section{Conclusion}

Increased clonal diversity in normal PJS mucosa

Mutation and selection are the twin driving forces of evolution. Epithelia may silently carry many potentially oncogenic mutations, such as p53 mutations, that only become exposed given the right context of conspiring genomic hits. Evolutionary change, therefore, is critically dependent on the presence of a range of heritable variants. Time to visible tumor formation is shortened in a niche that contains an increased number of progenitor cells [23]. An expanded progenitor pool thus reflects a cancer-prone state and predicts that pre-malignant lesions will arise at an accelerated pace in comparison to the general population. Our preliminary data on $C S X$ crypt methylation patterns in unaffected PJS mucosa are consistent with a protracted clonal evolution scenario in PJS. Longer crypt niche succesion times increase the level of genetic clonal diversity on which somatic evolution acting through natural selection depends (Fig. 5). Broadening the mutational repertoire present in the crypt increases the odds of fortuitiously incurring an accidental combination of mutations conferring a tumor phenotype. In this scenario, patients with PJS are expected to retain a greater number of mutations incurred at a normal background mutation rate. This increases genetic clonal diversity and accelerates somatic evolution in PJS crypt stem cell niches. Similar considerations concerning the neoplastic transformation in PJS apply to other epithelia with dedicated stem cell compartments (such as breast and pancreas), although we have focused in this paper on stem cell turnover in the gastrointestinal crypt. This lottery-like process of accumulating and selecting pathogenic hits in stem cell niches is played out in each of the approximately 15 million crypts that constitute the human colon.

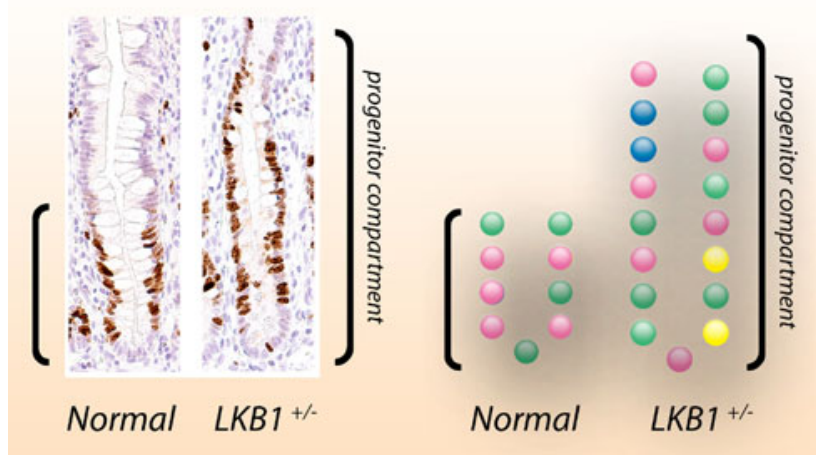

Fig. 5 Increased clonal diversity in PJS crypts. This diagram illustrates the consequences of differences in clonal evolution in stem cell compartments such as the intestinal crypt. Over time stem cell lineages accumulate mutations at a set background mutation rate. By using a polymorphic marker such as COX enzyme activity we can trace the appearance of individual lineages in the crypt (see Fig. 4). We have observed an expanded crypt progenitor zone in unaffected intestinal mucosa in PJS patients [34]. By comparing CSX crypt heterogeneity patterns in unaffected PJS mucosa and age-matched control crypts, we found that PJS crypts retain a greater number of polymorphic marker mutations. This indicates that clonal evolution is protracted in Peutz-Jeghers syndrome and predicts that pre-malignant lesions will arise at an accelerated pace in comparison to the general population (see text) 
Definitively testing the impact of $L K B 1$ hemizygosity in lineage succession in the intestinal stem cell compartment will require lineage tracing experiments where multiple individual lineages can be traced competing over time in individual crypts. With markers now available to unambiguously identify and genetically target progenitor cell populations [18] this crucial part of early tumor history has become open to scrutiny. In case $L K B 1$ hemizygosity can be shown to suffice for accelerated transformation then the hamartomatous epithelium covering the polyps would constitute the tip of the proverbial iceberg; it is the entire epithelial lining that is at increased risk for malignant degeneration. Demonstration of increased genetic clonal diversity in phenotypically normal Peutz-Jeghers mucosa would directly implicate a cancer-prone state at normal background mutation rates. This would provide important impetus to begin studying the impact of $L K B 1$ hemizygosity on lineage competition in stem cell niches. Thus, LKB1 hemizygosity may independently mediate polyp formation and the cancer-prone condition through one molecular mechanism played out on different timescales.

Open Access This article is distributed under the terms of the Creative Commons Attribution Noncommercial License which permits any noncommercial use, distribution, and reproduction in any medium, provided the original author(s) and source are credited.

\section{References}

1. Westerman AM, Entius MM, de Baar E, et al. (1999) PeutzJeghers syndrome: 78-year follow-up of the original family. Lancet 353(9160): 1211-1215

2. Katajisto P, Vallenius T, Vaahtomeri K et al (2007) The LKB1 tumor suppressor kinase in human disease. Biochim Biophys Acta 1775(1):63-75

3. McGarrity TJ, Amos C (2006) Peutz-Jeghers syndrome: clinicopathology and molecular alterations. Cell Mol Life Sci 63(18):2135-2144

4. Gao H, van Lier MG, Poley JW, Kuipers EJ, van Leerdam ME, Mensink PB (2010) Endoscopic therapy of small-bowel polyps by double-balloon enteroscopy in patients with Peutz-Jeghers syndrome. Gastrointest Endosc 71(4):768-773

5. Jansen M, de Leng WW, Baas AF et al (2006) Mucosal prolapse in the pathogenesis of Peutz-Jeghers polyposis. Gut 55(1):1-5

6. Singh B, Mortensen NJ, Warren BF (2007) Histopathological mimicry in mucosal prolapse. Histopathology 50(1):97-102

7. Miller LJ, Bartholomew LG, Dozois RR, Dahlin DC (1983) Adenocarcinoma of the rectum arising in a hamartomatous polyp in a patient with Peutz-Jeghers syndrome. Dig Dis Sci 28(11): 1047-1051

8. Shepherd NA, Bussey HJ, Jass JR (1987) Epithelial misplacement in Peutz-Jeghers polyps. A diagnostic pitfall. Am J Surg Pathol 11(10):743-749

9. Linos DA, Dozois RR, Dahlin DC, Bartholomew LG (1981) Does Peutz-Jeghers syndrome predispose to gastrointestinal malignancy? A later look. Arch Surg 116(9):1182-1184

10. Brugarolas J, Kaelin WG Jr (2004) Dysregulation of HIF and VEGF is a unifying feature of the familial hamartoma syndromes. Cancer Cell 6(1):7-10
11. Hemminki A, Tomlinson I, Markie D et al (1997) Localization of a susceptibility locus for Peutz-Jeghers syndrome to $19 p$ using comparative genomic hybridization and targeted linkage analysis. Nat Genet 15(1):87-90

12. Udd L, Katajisto P, Kyyronen M, Ristimaki AP, Makela TP. Impaired gastric gland differentiation in Peutz-Jeghers syndrome. Am J Surg Pathol 176(5):2467-2476

13. Huang X, Wullschleger S, Shpiro N, et al. (2008) Important role of the LKB1-AMPK pathway in suppressing tumourigenesis in PTEN deficient mice. Biochem J

14. Blanpain C, Horsley V, Fuchs E (2007) Epithelial stem cells: turning over new leaves. Cell 128(3):445-458

15. Barker N, van de Wetering M, Clevers H (2008) The intestinal stem cell. Genes Dev 22(14):1856-1864

16. Barker N, van Es JH, Kuipers J et al (2007) Identification of stem cells in small intestine and colon by marker gene Lgr5. Nature 449(7165):1003-1007

17. Schmidt GH, Winton DJ, Ponder BA (1988) Development of the pattern of cell renewal in the crypt-villus unit of chimaeric mouse small intestine. Development (Cambridge, England) 103(4):785-790

18. Snippert HJ, van der Flier LG, Sato T, et al. (2010) Intestinal crypt homeostasis results from neutral competition between symmetrically dividing Lgr5 stem cells. Cell 143(1):134-144

19. Lopez-Garcia C, Klein AM, Simons BD, Winton DJ (2010) Intestinal stem cell replacement follows a pattern of neutral drift. Science 330(6005):822-825

20. Klein AM, Nakagawa T, Ichikawa R, Yoshida S, Simons BD (2010) Mouse germ line stem cells undergo rapid and stochastic turnover. Cell Stem Cell 7(2):214-224

21. Doupe DP, Klein AM, Simons BD, Jones PH (2010) The ordered architecture of murine ear epidermis is maintained by progenitor cells with random fate. Develop Cell 18(2):317-323

22. Calabrese P, Tavare S, Shibata D (2004) Pretumor progression: clonal evolution of human stem cell populations. Am J Pathol 164(4):1337-1346

23. Kim KM, Calabrese P, Tavare S, Shibata D (2004) Enhanced stem cell survival in familial adenomatous polyposis. Am J Pathol 164(4):1369-1377

24. Raff RA (2007) Written in stone: fossils, genes and evo-devo. Nat Rev Genet 8(12):911-920

25. Yatabe Y, Tavare S, Shibata D (2001) Investigating stem cells in human colon by using methylation patterns. Proc Natl Acad Sci USA 98(19):10839-10844

26. Watts JL, Morton DG, Bestman J, Kemphues KJ (2000) The C. elegans par-4 gene encodes a putative serine-threonine kinase required for establishing embryonic asymmetry. Development (Cambridge, England) 127(7):1467-1475

27. Martin SG, St Johnston D (2003) A role for Drosophila LKB1 in anterior-posterior axis formation and epithelial polarity. Nature 421(6921):379-384

28. Jansen M, Ten Klooster JP, Offerhaus GJ, Clevers H (2009) LKB1 and AMPK family signaling: the intimate link between cell polarity and energy metabolism. Physiol Rev 89(3):777-798

29. Barnes AP, Lilley BN, Pan YA et al (2007) LKB1 and SAD kinases define a pathway required for the polarization of cortical neurons. Cell 129(3):549-563

30. Campbell F, Geraghty JM, Appleton MA, Williams ED, Williams GT (1998) Increased stem cell somatic mutation in the nonneoplastic colorectal mucosa of patients with familial adenomatous polyposis. Hum Pathol 29(12):1531-1535

31. Jishage K, Nezu J, Kawase Y et al (2002) Role of Lkb1, the causative gene of Peutz-Jegher's syndrome, in embryogenesis and polyposis. Proc Natl Acad Sci USA 99(13):8903-8908

32. Bardeesy N, Sinha M, Hezel AF et al (2002) Loss of the Lkb1 tumour suppressor provokes intestinal polyposis but resistance to transformation. Nature 419(6903):162-167 
33. Rossi DJ, Ylikorkala A, Korsisaari N et al (2002) Induction of cyclooxygenase- 2 in a mouse model of Peutz-Jeghers polyposis. Proc Natl Acad Sci USA 99(19):12327-12332

34. de Leng WW, Jansen M, Keller JJ et al (2007) Peutz-Jeghers syndrome polyps are polyclonal with expanded progenitor cell compartment. Gut 56(10):1475-1476
35. Clayton E, Doupe DP, Klein AM, Winton DJ, Simons BD, Jones PH (2007) A single type of progenitor cell maintains normal epidermis. Nature 446(7132):185-189

36. Udd L, Katajisto P, Rossi DJ et al (2004) Suppression of PeutzJeghers polyposis by inhibition of cyclooxygenase-2. Gastroenterology 127(4):1030-1037 